\title{
Effect of Retransmissions on the Performance of the IEEE 802.11 MAC Protocol for DSRC
}

Md. Imrul Hassan, Member, IEEE, Hai L. Vu, Senior Member, IEEE, Taka Sakurai, Member, IEEE, and Lachlan L. H. Andrew, Senior Member, IEEE

\begin{abstract}
We consider a dedicated short range communication (DSRC) network based on the IEEE 802.11 distributed coordination function (DCF) protocol for broadcasting vehicle-to-vehicle safety messages. Reliable delivery of broadcast safety messages to all nearby vehicles is an important issue, and is challenging due to the unreliable wireless links, lack of acknowledgments, fast changing network topology and the prevalence of hidden stations. This is especially important for so-called event messages that have a more stringent requirement for fast and guaranteed delivery than routine safety messages. In this paper, we propose two mechanisms, which can be implemented on top of standard DCF or EDCA, that use "blind" retransmissions with no feedback. We then develop a comprehensive analytical model to predict the behavior of the DCF protocol in unsaturated broadcast networks for both types of safety messages with and without retransmissions. In particular, we derive explicit expressions for the mean of the total packet delay and the packet delivery ratio (PDR). The model accuracy is validated using ns-2 simulations. We show that both the retransmission schemes improve the PDR of event messages, with acceptable degradation to that of routine safety messages.
\end{abstract}

\section{Index Terms}

DSRC, safety applications, performance analysis, retransmission, semi-reliable broadcast

\section{INTRODUCTION}

Dedicated Short Range Communication (DSRC) refers to the use of vehicle-to-vehicle and vehicle-toinfrastructure communications to improve road safety and increase transportation efficiency. Among the

Md. Imrul Hassan, Hai L. Vu and Lachlan L. H. Andrew are with Centre for Advanced Internet Architectures, Faculty of I.C.T., Swinburne Univ. of Technology, P.O. Box 218, VIC 3122, Australia.

Taka Sakurai is with Department of Electrical and Electronic Engineering, The University of Melbourne, VIC 3010, Australia. 
many candidate applications, cooperative collision avoidance (CCA) has attracted considerable interest in the research community as it can significantly improve road safety. In CCA, moving cars form a network to wirelessly communicate and warn each other of changing conditions or dangers ahead on the road to avoid accidents.

This can be achieved through communication between vehicles of both so-called routine and event messages [1]. The former typically contains information about vehicle state, such as position/direction and speed, and will be broadcast regularly by all vehicles. These messages constitute the majority of traffic load on the DSRC control channel and have a lifetime of a few seconds. The event messages, on the other hand, are triggered by events such as sudden braking.

These messages occur only occasionally, but can contribute significantly to the traffic load on the control channel when they do occur. Event messages have a more stringent requirement in terms of fast and guaranteed delivery, while routine messages may tolerate a higher packet error rate. To this end the DSRC medium access control (MAC) protocol has a vital role to play since both the reception and delay of safety messages are heavily affected by the MAC mechanism used for channel access.

Various MAC protocols have been proposed for vehicle-to-vehicle communications in the literature [2]. In this paper we focus on the IEEE 802.11p distributed coordination function (DCF) MAC protocol used in broadcast mode, and propose two alternate extensions to the basic protocol, namely sequential retransmission and batch retransmission, to improve the reliability of event message delivery. We develop accurate analytical models for DCF and the two retransmission extensions, and characterize the traffic regimes where the schemes offer gains. This is an extension of [3], in which the two proposals were proposed and evaluated by simulation. The contribution of the present paper is to develop analytical models for DCF and the two retransmission extensions used to broadcast in the presence of hidden stations. These are used to characterize the traffic regimes where the schemes offer gains.

DCF is a carrier sense multiple access (CSMA) protocol that was originally developed for wireless LANs. It has recently been adopted by the IEEE 802.11p standard [4] for DSRC applications. Although multi-hop transmissions could be used to enhance coverage, we will consider single-hop transmissions since that is sufficient in most situations to reach all vehicles in the vicinity of an event such as an accident [1].

Some of the technical attributes that make DCF suitable for vehicular-to-vehicle communications are that it is decentralized, it can operate with a variety of traffic loads, it does not require much reconfiguration upon a change in topology, and it supports both unicast and broadcast operating modes. The broadcast mode is more appropriate for time-critical applications like CCA because, unlike unicast, broadcast does 
not require the establishment of an association context between nodes before data communications can commence. The drawback of broadcast mode is that it is less reliable, since it cannot support any requestresponse handshaking procedures that typically improve reliability such as conventional acknowledgement or virtual carrier sensing (RTS/CTS), due to the risk of a "storm" of response packets. The CCA application typically requires a packet delivery ratio (PDR) of at least 90\% [5], and it has been suggested in [6] that the conventional DCF broadcast protocol may be unable to meet this requirement.

Several techniques to improve the reliability of DCF based on RTS/CTS have been proposed. One is to add additional state or message type information in the RTS/CTS mechanism for broadcast [7], [8]. This increases the complexity, but does not guarantee absolute reliability as is expected of RTS/CTS. A request-to-broadcast/clear-to-broadcast (RTB/CTB) scheme was proposed in [9]. To support reliable communication, the CTB packets are sent in the form of an in-band energy burst (a so-called black-burst or jamming signal [10]) whose length is proportional to the distance between the receiver and the sender. The protocol with RTB/CTB, however, is sensitive to topology changes. Another option is to emulate broadcast RTS/CTS by sending multiple unicast messages [11], but this incurs significant overhead. None of these can be implemented on top of standard IEEE 802.11p.

Another promising approach is to transmit the broadcast message multiple times [12], [13], [14]. Message repetition is either based on piggybacking of feedback for previous successful packet receptions [12], or carried out by an individual node randomly over the lifetime of a packet [14]. The piggybacked acknowledgement (PACK) protocol [13] essentially enables selective retransmission that promises high efficiency of channel use. We will compare the PDR performance of the piggybacked scheme and our proposed schemes in Section VIII.

To minimize protocol overhead, we study the use of retransmissions of event messages to improve reliability. We propose two retransmission schemes that neither rely on any feedback from the receiving nodes, nor introduce any additional protocol overhead, and are hence compatible with the DCF/EDCA protocol on which the DSRC standard is based. The first is a sequential retransmission scheme, in which every event message is retransmitted a fixed number of times and, to mitigate against repeat collisions, a backoff process with unchanging contention window is executed before each retransmission. Note that a similar approach has been proposed in [15] for non-standard MAC protocols. The second is a batch retransmission scheme, in which the event message and its fixed number of copies are sent back-to-back in a batch, once the node has gained access to the channel. Similarly to the sequential retransmission scheme, the batch scheme offers time diversity for an event message, but with the advantage of minimal additional delay. 
We develop an analytical model to predict the behavior of the DCF protocol in unsaturated broadcast networks with and without the retransmission schemes. We take special care to model the significant detrimental effect of hidden terminals. In the literature, the performance of unicast DCF has been extensively studied in the wireless LAN environment. Bianchi [16] analyzed the performance of a saturated network using a Markov chain model and Malone et al [17] extended the model to the unsaturated case. Tickoo and Sikdar [18] developed an alternative unsaturated model by modeling each node as a G/G/1 queue. There has been much less analytic work on broadcast, although see [19], [20]. Unlike our work, none of the above work considers hidden terminals (i.e., they assume a single collision domain).

Although there exists a body of literature analyzing the hidden terminal problem, there are several limitations of those models, as highlighted in [21] for the case of unicast communication. In [22], an analytical model is developed considering hidden nodes in broadcast/unicast communications. However, the model is based on measurements of the network which is not always feasible. The authors of [6] describe DSRC safety communications where broadcasting takes place in an unsaturated network with hidden terminals. The model in [6] assumes that the vulnerable period could be divided into a number of variable sized independent slots. This assumption, however, is not accurate when the average backoff duration is smaller than the vulnerable period itself [21]. In contrast to a single vulnerable period in the model of [6], we model the fact that the vulnerable period before a transmission is affected by CSMA, while that after transmission is not.

Note that [6] assumes that a backoff is initiated for each packet at a node, irrespective of whether the channel is idle or busy, which is a simplification of the 802.11 DCF. Finally, none of the existing analytical models capture the impact of possible retransmissions in the broadcast environment.

The rest of this paper is organized as follows. In Section II, we provide an overview of DSRC challenges and related standards. We then propose the two retransmission schemes to improve the reliability of event driven safety messages in Section III. In Section IV, we describe the system setup and assumptions used by our analytical model. The direct collision probability and the hidden terminal collision probability is calculated in Section V and Section VI respectively. In Section VII the mean and variance of the total delay is calculated. We verify the accuracy of our DCF model by comparison with simulation in Section VIII. Finally, we conclude the paper in Section IX.

\section{OVERVIEW OF THE DSRC PROTOCOL}

One of the goals of emerging DSRC systems is to enhance road safety by supporting the transmission of safety messages. Broadcast communication is appropriate for time-critical applications like road safety 
because, unlike unicast, broadcast does not require the establishment of an association context between nodes before data communications can commence. To enable preventative action, it is essential that safety messages are received correctly by surrounding vehicles in a timely fashion. Since the information is destined for all nearby vehicles, broadcasting is appropriate. However, as it is infeasible for all nodes to acknowledge broadcast data, data may be lost. This is especially true in the presence of hidden terminals. In the broadcast case, there are multiple receivers for each message, scattered in the transmission range of the sender, and any node that is within sensing range of any receiver but outside the transmission range of the sender is a potential hidden terminal.

The IEEE Wireless Access in Vehicular Environment (WAVE) project has recently published specifications for DSRC which includes the IEEE 802.11p standard [4] for the MAC and PHY layers. The 802.11p MAC protocol, like other 802.11 variants, uses the distributed coordination function (DCF) and the related Enhanced distributed channel access (EDCA) for channel access.

\section{A. IEEE 802.11 DCF Protocol Description}

In the IEEE 802.11 DCF, nodes contend for the channel using a carrier sense multiple access mechanism with collision avoidance (CSMA/CA). When a node has a packet to send, the channel must be sensed idle for a guard period known as the distributed interframe space, DIFS. If during that period of time, the channel becomes busy, then the access is deferred until the channel becomes idle again and a backoff process is initiated. Backoff intervals are slotted, and nodes are only permitted to commence transmissions at the beginning of slots. The discrete backoff time is uniformly distributed in the range $[0, W-1]$, where $W$ is called the contention window. The backoff time counter is decremented by one at the end of each idle slot. It is frozen when a packet transmission is detected on the channel, and reactivated after the channel is sensed idle again for a guard period. The guard period is equal to a DIFS if the transmitted packet was error-free, and equal to the extended interframe space, EIFS, if the packet was in error. The node transmits when the backoff counter reaches zero. A collision occurs when the counters of two or more nodes reach zero in the same slot. In broadcast mode, there is no ACK sent after the successful reception of a data packet, so the sender is unaware of any packet collision and there is no retransmission. After every data packet transmission, a node initiates a post-transmission random backoff.

EDCA differs from DCF by allowing stations a limited choice of the backoff window, interframe spaces and, importantly for our case, the number (TXOP) of packets transmitted when a contention is won. 


\section{Proposed Retransmission Schemes}

In order to improve the reliability of packet delivery for event messages, we propose two retransmission schemes, namely sequential retransmission and batch retransmission. The schemes are designed to be simple in order to maximize compliance with the 802.11p standard. In particular, both are "blind" retransmissions schemes in the sense that no feedback is required from the receivers - every event message is retransmitted a fixed number of times.

In the sequential scheme, every event message is retransmitted a fixed number of times separated by a standard backoff. The scheme is simple and compatible with the new standard, and hence can be easily deployed: when an emergency event occurs, instead of sending a single event message to the MAC layer, multiple copies are sent.

In the batch scheme, multiple copies of an event messages are transmitted back-to-back separated only by small inter-frame spaces (SIFS). This can be achieved using the TxOP feature of Enhanced distributed channel access (EDCA) mechanism. The only conditions under which all copies within a batch are corrupted are when it coincides with a batch of event messages from another node, or when the batch collides with multiple routine or event messages that are close together. The batch retransmission scheme has an advantage compared to the sequential scheme in that it reduces the number of collisions resulting in packet loss due to partial overlap of packets, reminiscent of the benefits of slotted Aloha over unslotted Aloha [23]. As a result, the loss probability will be considerably reduced. The delay experienced by retransmissions in this schemes is also expected to be negligible. Note that the TxOP option of the EDCA mechanism has been explicitly removed from ad hoc operation in the current version of the standard. We argue that its performance poses an attractive alternative for $\mathrm{V} 2 \mathrm{~V}$ system design and configuration, and that it should be allowed as it is in regular 802.11.

One of our key assumptions is that a packet cannot be successfully decoded if any part of it experiences a collision with a packet transmitted by another node within sensing range of the receiver. A major cause of collisions is the presence of hidden nodes which are unaware of the sender. As a result, the combined effect of other nodes on the channel from the perspective of a given sending node, can be viewed as a bursty on-off stochastic interference process, where transmission during the "on" period results in collision. Our two retransmission schemes aim to exploit time diversity in different ways to improve the likelihood that at least one copy of an event packet is transmitted during an "off" period of the interference process. We arbitrarily select any vehicle and denote it as the "tagged node", and define its location to be the origin. We also consider an arbitrary packet transmitted by the tagged node as the 
"tagged packet" and calculate the performance metrics for that packet only. In the sequential scheme, this is achieved by transmitting multiple copies back-to-back at the application layer. This randomizes the gaps between retransmissions by executing a backoff process with unchanging contention window $W$ before each retransmission. The units of the backoff period are of the order of a packet length.

\section{SYSTEM SETUP}

Let us consider a scenario of vehicle-to-vehicle communications for CCA applications on a highway. The highway consists of several lanes with vehicles moving in both directions. Define the carrier sense (CS) range to be some region containing the tagged node. In our model we make the following assumptions which will be discussed after stating them.

A.1 For each node, there are $N_{t r}$ nodes within the CS range whose transmissions it can sense and collide with, and $N_{p h}$ "hidden" nodes whose transmissions it cannot sense but with whose transmissions its will collide.

A.2 If a packet transmission by a node overlaps in time with one by any of its $N_{t r}+N_{p h}$ nodes, then both packets will be irretrievably corrupted for all receivers. Otherwise, the packet is received correctly.

A.3 The transmission attempts of each node are independent of those from other nodes, except for the effect of carrier sensing. Moreover, the collision probability and probability of an arriving packet observing the channel busy are both constant regardless of the node's state.

A.4 All nodes generates event packets according to independent Poisson processes, each with rate $\alpha \lambda$ [packets/sec] with $\alpha \in[0,1]$, and generate routing safety messages as independent Poisson processes with rate $(1-\alpha) \lambda$.

A.5 If a packet arrives when the channel is idle, it will not suffer a collision with any node within the carrier sense range.

A.6 The probability of a given transmission colliding with more than one other transmissions is negligible, where a burst of transmissions is treated as a single transmission.

Assumption A.1 models the case that vehicles are distributed uniformly along a highway, which is narrow compared to the sensing range. In the numerical results, we will assume that vehicles form a homogeneous Poisson processes, and there is a carrier sense radius $R$ such that any vehicle in the range $[R, 2 R]$ and $[-2 R,-R]$ of a selected (tagged) vehicle is a potential hidden terminal. Let $\beta$ be a vehicle density in vehicles per meter on the highway. The average number of vehicles in the transmission range 
of the tagged vehicle including the tagged node is $N_{t r}=1+2 \beta R$, and the average number of vehicles in the potential hidden terminal area is $N_{p h}=2 \beta R$.

Assumption A.2 implies that there is no capture effect, and that the error correcting codes used are insufficient to recover packets from collisions. Also, we consider only collision related packet losses, and not those due to channel error. So, a node within the carrier sense range of the transmitter can reliably decode the message if no collision occurs. The model can be extended to include imperfect channel conditions as explained later in the discussion following (27)-(31).

Assumption A.3 and the Poisson nature of A.4 are common in performance studies of the MAC protocol in mobile wireless ad-hoc networks [6] and help make the model analytically tractable, while still yielding meaningful indications of MAC performance.

Assumption A.3 also classifies the packets into event packets and routine safety packets. Under the proposed mechanisms, each event packet is transmitted $m$ times; under the sequential scheme a random backoff is performed between each of these transmissions, while under the batch scheme the retransmissions are only separated by SIFS. Furthermore, since it is a second-order effect, we ignore any possible residual post-backoff time [21] from the previously transmitted packet when modeling the delay of a packet newly arrived to the transmit buffer of a node.

DSRC event messages are bursty; emergency events such as an animal running across a road will cause a surge of event messages from multiple vehicles. In this paper, we model the period after an emergency event has occurred when the chance of packet drop is the highest. It is within this short interval that we apply a Poisson model. This model captures the phenomenon of having a "rate" of event messages, without assuming them to be periodic. The Poisson nature of the arrival process is not disturbed by MAC effects, since the time scale of milliseconds for message delivery (see Section VIII) is small compared with the duration of the surge (of the order of a human reaction time, around $1 \mathrm{~s}$ [24]). Moreover, events such as hard braking, which may be expected to have high correlation, actually have weak correlation between vehicles [25]. Such weak correlation between many agents naturally leads to a Poisson process. The model uses this assumption in two places; at each place, we explain how the model would differ if this assumption were relaxed.

To appreciate assumption A.5, recall that when a packet arrives and finds the channel idle for DIFS period, the vehicle immediately sends the packet without performing a backoff. In this case, a collision can occur only when another packet is generated at some other vehicle within the propagation delay. As the propagation delay in the studied transmission range is negligible, we ignore any collisions of this type. 
Assumption A.6 arises since multiple transmissions within a short period are a second-order effect when the load is relatively light. This assumption makes our results optimistic when the load is high, especially for batch retransmissions.

Our objective is to develop an approximation to compute the collision probability and the packet delay experienced by the tagged vehicle. To this end, the fixed point approximation is established by combining the set of equations for the collision probability expressed in terms of the mean service time experienced by each packet sent by the tagged node, with an opposing set of equations for the mean service time expressed in terms of the collision probability. We derive the former set of equations in Section V and the latter in Section VII.

In the following derivations, many quantities are distinguished by subscripts and superscripts. For brevity, we use notation of the form $A_{j \in J}=f(j)$ to mean that $A_{j}=f(j)$ for each $j \in J$.

\section{Direct Collision Probability}

In this section, we derive the probability of a "direct" collision (i.e., one with a node within the CS range), for both sequential transmission scheme and batch transmission scheme, and in the next subsection, we extend the model to allow for hidden terminals.

\section{A. Standard DCF}

Since we ignore direct collisions of packets which do not perform a backoff, we first calculate the probability of performing a backoff and then calculate the collision probability for the first transmission attempt.

Let $\tau$ be the probability that a vehicle attempts to transmit in an arbitrary slot given that it has a packet in the queue. This probability is

$$
\tau=\frac{1}{(\bar{W}+1)}
$$

where $\bar{W}=(W-1) / 2$ is the average number of backoff slots preceding a transmission. In contrast to the fixed point of Bianchi [16], $\tau$ is not part of the current fixed point because nodes never retransmit.

Define $\rho$ as the fraction of time the vehicle has a packet to send. Then

$$
\rho=\min (\lambda E[S], 1)
$$

where $E[S]$ is the average time to serve a packet, to be derived in Section VII. We only consider the case that $\rho<1$. 
Let $b$ denote the probability that the channel is sensed busy when a new packet arrives. A backoff is performed prior to transmitting a packet if the packet arrived to a non-empty buffer or if the packet arrived when the channel was busy. Since the system is a single-server queue, the probability that a packet that arrives to the buffer finds the buffer empty is given by $1-\rho$. Therefore, by the assumption A.3, the probability that the packet undergoes a backoff is $1-(1-\rho)(1-b)$.

We calculate the busy probability, $b$ by quantifying the portion of time the channel is busy due to transmissions. We have $N_{t r}-1$ vehicles other than the tagged vehicle transmitting $\lambda$ [packets/sec]. Also, we define $T$ as the complete transmission time of a packet including DIFS,

$$
T=t_{d a t a}+t_{d i f s}
$$

If there is no collision, then all the packet transmissions in the CS range should take a fraction $\mathcal{T}=\left(N_{t r}-1\right) \lambda T$ of the time. However, when a collision occurs, exactly two nodes are involved (assumption A.6), and their two transmissions coincide exactly due to carrier sensing. Thus, the transmission time to send the packets which collide is reduced by $\left(N_{t r}-1\right) \lambda d / 2 T$ where $d$ is the probability that a packet is involved in a direct collision. Subtracting this from $\mathcal{T}$ gives

$$
b=\left(N_{t r}-1\right) \lambda(1-d / 2) T .
$$

We now calculate $d$. After the backoff of the tagged vehicle is completed, the tagged vehicle sends the packet in the following slot. If another vehicle sends a packet at the same slot as the tagged vehicle, a collision occurs and the packet is lost. For any vehicle other than the tagged vehicle, the probability of transmitting in slot in which the tagged node is transmitting is $\rho \tau$. A collision occurs when any of the $N_{t r}-1$ vehicles transmit in the same slot as the tagged vehicle given that the tagged vehicle performs the backoff. Thus, the direct collision probability $d$ is

$$
d=(1-(1-\rho)(1-b))\left(1-(1-\rho \tau)^{N_{t r}-1}\right) .
$$

\section{B. Proposed extensions}

The direct collision probabilities for the sequential and batch schemes are similar to the above.

Let $b^{s}$ and $b^{b}$ represent the probability that the channel is sensed busy for the sequential and batch schemes respectively. Also, let $d_{j}^{i}$ denote the direct collision probabilities for routine $(j=r)$ and event $(j=e)$ packets under the sequential $(i=s)$ and batch $(i=b)$ retransmission schemes. Since routine packets are treated the same by both schemes, we will first consider $d_{r}^{i \in\{s, b\}}$. 
1) Routine packets: The direct collision probability for routine packets is similar to Equation (5).

$$
d_{r}^{i \in\{s, b\}}=\left(1-(1-\rho)\left(1-b^{i}\right)\right)\left(1-(1-\rho \tau)^{N_{t r}-1}\right) .
$$

The probability of losing an event packet due to direct collisions differs for the two schemes, which will now be considered in turn.

2) Event packets: Sequential transmissions: For the sequential scheme, the first transmission of an event packet is equivalent to a routine packet, and so the direct collision probability for the first attempt of event packets, denoted by $d_{e, 0}^{s}$, is

$$
d_{e, 0}^{s}=d_{r}^{s}=\left(1-(1-\rho)\left(1-b^{s}\right)\right)\left(1-(1-\rho \tau)^{N_{t r}-1}\right),
$$

where $b^{s}$ will be calculated shortly. For any subsequent attempts of the event packet for the sequential scheme, we note that there is always a backoff preceding the packet transmission. As a result the slots are synchronized among the neighboring nodes and any attempt to transmit multiple packets in the same slot results in a collision. So, letting $d_{e, i}^{s}$ denote the probability of direct collision in the $i$ th attempt of an event packet for the sequential scheme, we have for $i=1, \ldots, m-1$,

$$
d_{e, i}^{s}=\left(1-(1-\rho \tau)^{N_{t r}-1}\right) .
$$

Since the probabilities in (7) and (8) are independent by assumption A.3, the probability of a packet being undeliverable is

$$
\begin{aligned}
d_{e}^{s} & =d_{e, 0}^{s} \prod_{i=1}^{m-1} d_{e, i}^{s} \\
& =\left(1-(1-\rho)\left(1-b^{s}\right)\right)\left(d_{e, 1}\right)^{m} .
\end{aligned}
$$

In order to calculate the fraction of time the channel is busy, consider the mean direct collision probability,

$$
\begin{aligned}
\bar{d}_{e}^{s} & =\frac{d_{e, 0}^{s}+\sum_{i=1}^{m-1} d_{e, i}^{s}}{m} \\
& =\left(1-\frac{(1-\rho)\left(1-b^{s}\right)}{m}\right) d_{e, 1}^{s} .
\end{aligned}
$$

Here $b^{s}$ is the probability that the channel is sensed busy when a new packet arrives for the sequential scheme,

$$
b^{s}=\left(N_{t r}-1\right) \lambda\left((1-\alpha)\left(1-d_{r}^{s} / 2\right)+m \alpha\left(1-\bar{d}_{e}^{s} / 2\right)\right) T^{s}
$$


where $T^{s}$ is the complete transmission time of a packet including the DIFS period for the sequential scheme,

$$
T^{s}=t_{\mathrm{data}}+t_{\mathrm{difs}}
$$

Equation (11) is based on quantifying the portion of time the channel is busy due to transmissions. We have $N_{t r}-1$ vehicles in the CS range other than the tagged vehicle, transmitting $(1-\alpha) \lambda$ [packets/sec] for routine safety packets and $\alpha \lambda$ [packets/sec] with $m$ transmission attempts per packet for event packets. If there is no collision, then all the packet transmissions should take $\mathcal{T}=\left(N_{t r}-1\right) \lambda((1-\alpha)+m \alpha) T^{s}$ time each second. However, when a collision occurs, exactly two nodes are involved (Assumption A.6), and their two transmissions coincide exactly due to carrier sensing. Thus, the transmission time to send the packets which collide is reduced by $\left(N_{t r}-1\right) \lambda\left((1-\alpha) d_{r}^{s} / 2+(m \alpha) \bar{d}_{e}^{s} / 2\right) T^{s}$. Subtracting this from $\mathcal{T}$ gives (11).

Equations (7)-(11) will be combined with $\rho$ calculated in Section VII to form a fixed-point equation.

3) Event packets: Batch retransmission: To calculate the direct collision probability of the event packets for the batch scheme, note that a single routine packet can overlap with at most two copies of the event packet. This means that, under assumption A.6, if $m \geq 3$ then an event packet can always be received unless it collides with another event packet. As the rate of arrival of event packets is $\alpha \lambda$, the direct collision probability of event packets under the batch scheme can be shown, similarly to (6), to be

$$
d_{e}^{b}=\left(1-(1-\rho)\left(1-b^{b}\right)\right)\left(1-(1-\alpha \rho \tau)^{N_{t r}-1}\right) .
$$

Like in the sequential case, the probability that the channel is sensed busy when a new packet arrives for the batch scheme is

$$
b^{b}=\left(N_{t r}-1\right) \lambda\left((1-\alpha)\left(1-d_{r}^{b} / 2\right) T_{r}^{b}+\alpha\left(1-d_{e}^{b} / 2\right) T_{e}^{b}\right),
$$

where $T_{r}^{b}$ and $T_{e}^{b}$ are the complete transmission time of a packet including the DIFS period for routine and event packets respectively given by

$$
\begin{aligned}
& T_{r}^{b}=t_{\mathrm{data}}+t_{\mathrm{difs}}, \\
& T_{e}^{b}=m t_{\mathrm{data}}+(m-1) t_{\mathrm{sifs}}+t_{\mathrm{difs}} .
\end{aligned}
$$

\section{HidDEn Terminal Collision}

In the previous section, we obtained the probability of collisions among $N_{t r}$ terminals within the sensing range. Now we present an approach to calculate the PDR including the influence of hidden terminals. The PDR does not form part of the fixed point, but is one of the primary performance measures of interest. 


\section{A. Standard DCF}

For a tagged packet to be successfully received, both of the following must occur. First, when the tagged vehicle starts its transmission, none of the hidden terminals can be in what we call the transmitting state; we call this event $H^{b}$ ("hidden, before"). A hidden terminal is said to be in the transmitting state if it is either transmitting a packet or deferring for a DIFS period associated with an imminent packet transmission. (This condition will be relaxed for event packets in the batch case.) Second, after the tagged vehicle starts its transmission given $H^{b}$, none of the hidden terminals should start transmitting until after the tagged vehicle is finished; we call this conditional event $H^{a}$ ("hidden, after") for the first transmission attempt.

By a similar argument as used to derive (4), the probability of finding all hidden terminals in the non-transmitting state is

$$
P\left(H^{b}\right)=1-N_{p h} \lambda(1-d / 2) T
$$

For event $H^{a}$ we need to calculate the probability that a packet is generated by the hidden terminal after the tagged vehicle starts its transmission and eventually collides with the transmission of the tagged vehicle. The combined packet arrival process from all the hidden terminals is a Poisson process and the total number of transmission attempts per second is $\lambda N_{p h}$. Condition $H_{r}^{a}$ is met if no packet is generated at any of the hidden terminals during the $t_{\mathrm{data}}-t_{\mathrm{difs}}$ period. The event probabilities are then

$$
P\left(H^{a}\right)=\exp \left(-N_{p h} \lambda\left(t_{\mathrm{data}}-t_{\mathrm{difs}}\right)\right)
$$

This, and similar calculations below, is the first place where the Poisson assumption is used. Note that any arrival process with a well-defined "current rate" of $N_{p h} \lambda$, even if not Poisson, will yield the same first-order approximation of

$$
P\left(H^{a}\right) \approx 1-N_{p h} \lambda\left(t_{\mathrm{data}}-t_{\mathrm{difs}}\right)
$$

here, which is applicable when $N_{p h} \lambda\left(t_{\text {data }}-t_{\text {difs }}\right) \ll 1$.

We will now consider these two events for the batch and sequential schemes separately.

\section{B. Proposed extensions}

1) Sequential retransmission: For the sequential scheme we denote the first condition, $H^{\cdot, b}$, as $H_{r}^{s, b}$ for routine safety messages and as $H_{e, i}^{s, b}$ for the $i$ th attempt $(i=0,1, \ldots, m-1)$ of an event messages. Note that only event packets are sent multiple times and collision in the first attempt has the same probability 
as the collision for routine safety packets. By a similar argument as used to derive (11), the probability of finding all hidden terminals in the non-transmitting state is

$$
P\left(H_{r}^{s, b}\right)=P\left(H_{e, 0}^{s, b}\right)=1-N_{p h} \lambda\left((1-\alpha)\left(1-d_{r}^{s} / 2\right)+m \alpha\left(1-\bar{d}_{e}^{s} / 2\right)\right) T^{s} .
$$

Event $H^{\cdot, a}$ is again specialized to $H_{r}^{s, a}$ for a routine safety packet and $H_{e, i}^{s, a}$ for an event packet in its $i$ th attempt. By a similar process as used to derive (18), the probability of no hidden packets transmitting after the tagged transmission starts is

$$
P\left(H_{r}^{s, a}\right)=P\left(H_{e, 0}^{s, a}\right)=\exp \left(-N_{p h} \lambda((1-\alpha)+m \alpha)\left(t_{\text {data }}-t_{\text {difs }}\right)\right) .
$$

For notational convenience, let $H_{e, 0}^{s}=H_{e, 0}^{s, b} \wedge H_{e, 0}^{s, a}$ be the event that the first attempt of a tagged event packet does not collide with any packets from a hidden terminal, sent either before or after itself.

It remains to calculate the probability for hidden terminal collision in the subsequent transmission attempts for the sequential scheme given that hidden collisions occurred for all the previous attempts. Note that hidden collisions in subsequent attempts are not independent. ${ }^{1}$ If a collision occurs in the first attempt where the colliding packet is not in its last transmission attempt, subsequent transmission attempts are more likely to result in collisions. We need to calculate the probability of a subsequent collision, $p_{s u b}$, given that the first attempt resulted in a collision due to a hidden terminal and the other packet is not in its last attempt.

Let the two nodes in the collision be denoted $u$ and $v$, and let $u$ be the one that transmitted first. Consider the (usual) case that $u$ also starts its retransmission before $v$ finishes. By the assumption A.6, a collision will occur if and only if $v$ 's retransmission starts before $u$ 's retransmission finishes. Let $t$ be the time at which $u$ 's previous transmission finishes. The expected time for $u$ 's retransmission to finish is $t+$ $t_{\text {data }}+\bar{W} \mathrm{E}[Y]$, where $Y$ is the random duration of a backoff slot, calculated in Section VII. The expected time for $v$ 's next backoff period to start is $t_{\text {data }} / 2$. Hence, the collision probability on the subsequent attempt can be approximated by the probability that $v$ 's backoff lasts less than $t_{\text {data }} / 2+(W-1) \mathrm{E}[Y] / 2$. This backoff is $\mathrm{E}[Y]$ multiplied by an integer uniformly distributed on $[0,(W-1)]$, if $W$ is large enough that the ensemble average $\mathrm{E}[Y]$ is experienced. Hence the probability of a collision on the subsequent attempt can be modeled as

$$
p_{\text {sub }}=\min \left(\frac{t_{\mathrm{data}} / 2+(W-1) \mathrm{E}[Y] / 2}{W \mathrm{E}[Y]}, 1\right) .
$$

\footnotetext{
${ }^{1}$ This is not strictly in keeping with the Poisson assumption A.4
} 
The probability that the other packet is not in its last attempt is $\frac{m-i}{m}$ for the $i$ th retransmission. We assume that the probability of collision due to all other hidden nodes can be calculated as previously. The probability that none of them collide is then

$$
\begin{aligned}
& P\left(H^{\prime}\right)=\exp (\left.-\left(N_{p h}-1\right)^{+} \lambda((1-\alpha)+m \alpha)\left(t_{\mathrm{data}}-t_{\mathrm{difs}}\right)\right) \\
&\left(1-\left(N_{p h}-1\right)^{+} \lambda\left((1-\alpha)\left(1-d_{r}^{s} / 2\right)+m \alpha\left(1-\bar{d}_{e}^{s} / 2\right)\right) T^{s}\right),
\end{aligned}
$$

where $(x)^{+}=\max (0, x)$. As such, the probability of no hidden collision for the subsequent attempts given that hidden collisions occurred on all previous attempts is

$$
P\left(H_{e, i}^{s}\right)=P\left(H^{\prime}\right)\left(1-\frac{m-i}{m} p_{s u b}\right) .
$$

2) Batch retransmissions: Consider first the "before" event, $H^{\bullet, b}$. Following a similar argument to the derivation of (11), we can show that the probability of event $H^{\cdot, b}$ for routine safety packets under the batch scheme, denoted $H_{r}^{b, b}$, is

$$
P\left(H_{r}^{b, b}\right)=1-N_{p h} \lambda\left((1-\alpha)\left(1-d_{r}^{b} / 2\right) T_{r}^{b}+\alpha\left(1-d_{e}^{b} / 2\right) T_{e}^{b}\right) .
$$

For event packets, condition $H^{\cdot, b}$ can be relaxed; the packet is retransmitted multiple times back to back and lost only when it collides with the first transmission of another event packet since otherwise the last transmission of the tagged packet will succeed, by the assumption A.6 that each packet collides with only one other. The probability of the "before" condition for event packet, $H_{e}^{b, b}$, is thus

$$
P\left(H_{e}^{b, b}\right)=1-N_{p h} \alpha \lambda\left(1-d_{e}^{b} / 2\right)\left(t_{\text {data }}+t_{\text {difs }}\right) .
$$

For event $H^{\cdot, a}$ we need to calculate the probability that a packet is generated by the hidden terminal after the tagged vehicle starts its transmission and eventually collides with the transmission of the tagged vehicle. Again, there are two cases, $H_{r}^{b, a}$ and $H_{e}^{b, a}$, when the tagged packet is a routine or event packet, respectively. The combined packet arrival process from all the hidden terminals is a Poisson process and the total number of transmission attempts per second is $\lambda N_{p h}$ for the batch scheme. Condition $H_{r}^{b, a}$ is met if no packet is generated at any of the hidden terminals during the period of length $t_{\text {data }}-t_{\text {difs }}$. For event packets, $H_{e}^{b, a}$ should only consider collisions with event packets within the first transmission attempt, which form a Poisson process with rate $\alpha \lambda$. The event collision probabilities are then

$$
\begin{aligned}
& P\left(H_{r}^{b, a}\right)=\exp \left(-\lambda N_{p h}\left(t_{\mathrm{data}}-t_{\mathrm{difs}}\right)\right) \\
& P\left(H_{e}^{b, a}\right)=\exp \left(-\alpha \lambda N_{p h}\left(t_{\mathrm{data}}-t_{\mathrm{difs}}\right)\right)
\end{aligned}
$$




\section{Packet delivery ratios}

To calculate the packet delivery ratio, we approximate direct collisions and collisions due to hidden terminals as independent, and note that a packet is dropped when all the transmission attempts fail. Thus, the PDR for DCF and for both type of packets under both schemes can finally be expressed as

$$
\begin{aligned}
P D R & =(1-d) P\left(H^{b}\right) P\left(H^{a}\right) \\
P D R_{r}^{s} & =\left(1-d_{r}^{s}\right) P\left(H_{r}^{s, b}\right) P\left(H_{r}^{s, a}\right) \\
P D R_{e}^{s} & =\left(1-d_{e}^{s}\right)\left(1-\prod_{i=0}^{m-1}\left(1-P\left(H_{e, i}^{s}\right)\right)\right) \\
P D R_{r}^{b} & =\left(1-d_{r}^{b}\right) P\left(H_{r}^{b, b}\right) P\left(H_{r}^{b, a}\right) \\
P D R_{e}^{b} & =\left(1-d_{e}^{b}\right) P\left(H_{e}^{b, b}\right) P\left(H_{e}^{b, a}\right)
\end{aligned}
$$

These equations could be modified to model a channel with packet errors with probability $\epsilon$. The right hand sides of (27), (28), and (30) would be multiplied by $(1-\epsilon)$, and in (29), $P\left(H_{e, i}^{s}\right)$ would become $(1-\epsilon) P\left(H_{e, i}^{s}\right)$. The form of (31) would become more complex, as it would depend on the number of packets that avoid collision, rather than simply whether that number is non-zero. However, such an extension is out of the scope of this paper.

\section{ViI. Delay Calculation}

In this section, we derive an expression for the packet delay using probabilistic arguments. The total delay (or sojourn time) experienced by a packet of a tagged vehicle includes the complete time to transmit the packet, the access delay and the waiting time of the packet in the transmit node's queue, if any. The access delay of a packet is defined as the time interval between the instant the packet reaches the head of the queue, to the instant when the last attempt of the packet transmission starts. Thus, the access delay consists of the backoff periods associated with all the transmission attempts and the time of all the collisions of that packet. We denote the total delay of the packet under retransmission scheme $i \in\{s, b\}$ by $D^{i}$, given by

$$
D^{i \in\{s, b\}}=Q^{i}+S^{i}
$$

where $Q^{i \in\{s, b\}}$ are random variables (r.v.'s) representing the queueing delay, and $S^{i \in\{s, b\}}$ is the service time, during which the MAC protocol is attempting to transmit the packet.

Remark 1: The delay experienced by the application is the time until the packet is first received successfully. The "total delay" $D^{i \in\{s, b\}}$ is an over-estimate of this "application delay", which is quite conservative if the probability of success is high, especially for the sequential scheme. 


\section{A. Distribution of service time}

To calculate the distribution of the service times under both of the proposed schemes, we first define some terms.

For each packet transmission in the sequential scheme, the channel is occupied for the duration of the actual packet transmission $\left(t_{\text {data }}\right)$ and one DIFS; recall that we define the complete transmission time $T^{s}$ as the sum of the actual packet transmission time and one DIFS period. The complete transmission time $T_{r}^{b}$ for routine safety packets in the batch scheme is also the sum of the actual packet transmission time and one DIFS period; however, for event packets the complete transmission time $T_{e}^{b}$ consists of one DIFS, actual transmission time for all the attempts and SIFS periods in between transmissions. We also define the service time of the queue $S$ as the sum of the access delay $A$ and the transmission delay $T$. The service times for the sequential and batch schemes depend on whether the packet is an event packet or a routine safety packet, and can be expressed as

$$
S^{i \in\{s, b\}}=\left\{\begin{array}{lll}
A_{r}^{i}+T_{r}^{i} & \text { w.p. } & 1-\alpha \\
A_{e}^{i}+T_{e}^{i} & \text { w.p. } & \alpha
\end{array}\right.
$$

where $A_{j \in\{r, e\}}^{i \in\{s, b\}}$ are the access delays. and the notation "w.p." stands for "with probability".

To determine the access delay, we identify three scenarios that can confront a newly-generated packet in a vehicle operating in an unsaturated network as follow

1) A packet arrives to an empty buffer and finds the channel idle with probability $(1-\rho)(1-b)$. The access delay for the first attempt in this case is zero as the tagged vehicle transmits the packet without any backoff.

2) A packet arrives to an empty buffer but finds the channel busy with probability $(1-\rho) b$. The vehicle must wait until the ongoing transmission is finished and then perform a backoff before transmitting the packet.

3) A packet arrives to a non-empty buffer with probability $\rho$ and when it reaches the head of the queue, a backoff is performed before transmitting the packet.

Now, for the sequential scheme event packets are sent multiple times with a backoff in between each transmission attempt. Routine safety packets for both scheme are sent only once and also multiple transmission attempts of an event packet for the batch transmission scheme can be considered as a single transmission attempt with larger duration. In the cases $(i, j) \in\{(s, r),(b, r),(b, e)\}$, there is a single 
transmission attempt, whose access delay is

$$
A_{j}^{i}=\left\{\begin{array}{lll}
0 & \text { w.p. } & (1-\rho)\left(1-b^{i}\right), \\
B_{j}^{i}+T_{\text {Res }}^{i} & \text { w.p. } & (1-\rho) b^{i}, \\
B_{j}^{i} & \text { w.p. } & \rho,
\end{array}\right.
$$

where $T_{R e s}^{i \in\{s, b\}}$ is the residual time of the packet using the medium when the tagged packet arrives, and $B_{j}^{s}$ and $B_{j}^{b}$ represent the total backoff duration including periods when the backoff counter is suspended. For sequential retransmissions, all packets have length $T^{s}$ and so $T_{\text {Res }}^{s}$ is uniformly distributed on $\left(0, T^{s}\right)$. For batch transmissions, the remaining time depend on the probability that the arrival occurs during an event packet, and satisfies

$$
T_{R e s}^{b} \sim\left\{\begin{array}{llc}
\mathcal{U}\left(0, T_{e}^{b}\right) & \text { w.p. } & \frac{\alpha T_{e}^{b}}{\alpha T_{e}^{b}+(1-\alpha) T_{r}^{b}} \\
\mathcal{U}\left(0, T_{r}^{b}\right) & \text { w.p. } & \frac{(1-\alpha) T_{r}^{b}}{\alpha T_{e}^{b}+(1-\alpha) T_{r}^{b}}
\end{array}\right.
$$

where $\mathcal{U}(a, b)$ denotes a uniform random variable on the interval $(a, b)$.

Using sequential retransmissions, the delay for the first attempt of an event packet is also the same as above. However, each subsequent transmission attempt follows a backoff period. So, the total access delay for event packets in the sequential scheme can be defined as

$$
A_{e}^{s}=A_{r}^{s}+\sum_{n=1}^{m-1}\left(B_{e, n}^{s}+T^{s}\right),
$$

where, $m$ is the number of transmission attempts for each of the packets and $\left\{B_{e, n}^{s}\right\}$ is a sequence of i.i.d. r.v.s representing the backoff duration for each transmission attempt.

During the backoff process, every slot can be interrupted by successful transmissions or collisions of packets transmitted by other vehicles. During the interruption, the backoff counter is suspended and when the backoff counter is resumed, it starts from the beginning of the interrupted slot. Thus, we can express $B$ as a random sum

$$
B=\sum_{n=1}^{U} Y_{n}
$$

where $\left\{Y_{n}\right\}$ is a sequence of i.i.d. r.v.s. representing the duration of each slot, and $U$ is the backoff counter value which is uniformly distributed in the range $[0, W-1]$.

If no other vehicle transmits in a given slot then $Y=\sigma$, where $\sigma$ is defined to be the duration of an idle backoff slot. If one or more vehicles transmit in that slot, then the tagged vehicle will suspend its backoff process for the duration of the complete transmission, $T$. Recall that, the probability that a vehicle attempts to transmit in an arbitrary slot given that it has a packet in its buffer is given by $\tau$ and 
the probability that the buffer is non-empty is $\rho$. Therefore, the probability of a vehicle transmitting in an arbitrary slot is $\rho \tau$ and a backoff slot of the tagged vehicle is interrupted when any of the other $N_{t r}-1$ vehicles transmit in that slot with probability $1-(1-\rho \tau)^{N_{t r}-1}$. Now, for the sequential scheme both routine and event packets have the same transmission time, $T^{s}$. Therefore, $Y^{s}$ for the sequential scheme is

$$
Y^{s}=\left\{\begin{array}{lll}
\sigma & \text { w.p. } & (1-\rho \tau)^{N_{t r}-1}, \\
\sigma+T^{s} & \text { w.p. } & 1-(1-\rho \tau)^{N_{t r}-1}
\end{array}\right.
$$

where $1-(1-\rho \tau)^{N_{t r}-1}$ is the probability that a slot is busy due to transmissions by other vehicles.

For batch transmission, the slot period is $Y=\sigma$ if no interruption occurs, it can be $\sigma+T_{r}^{b}$ if the slot is interrupted by a routine packet, or it can be $\sigma+T_{e}^{b}$ if the slot is interrupted by an event packet. Since collisions are rare, we estimate the slot period given a transmission as $T_{e}^{b}$ with probability $\alpha$ and $T_{r}^{b}$ otherwise. We can calculate $Y_{e}^{b}$ and $Y_{r}^{b}$ for the batch scheme similar to (38) as

$$
Y_{j \in\{e, r\}}^{b}=\left\{\begin{array}{lll}
\sigma & \text { w.p. } & (1-\rho \tau)^{N_{t r}-1}, \\
\sigma+T_{e}^{b} & \text { w.p. } & \alpha\left(1-(1-\rho \tau)^{N_{t r}-1}\right), \\
\sigma+T_{r}^{b} & \text { w.p. } & (1-\alpha)\left(1-(1-\rho \tau)^{N_{t r}-1}\right) .
\end{array}\right.
$$

These equations determine the distribution of the access delay.

\section{B. Mean and Variance of Delay}

The mean total delay of a packet is an important performance metric. In addition, the service time is needed to obtain the fixed point for $\rho$ in (2). In this section, we determine the mean and variance of the service time and the mean of the total delay. We express them using means and variances of the constituent random variables.

First, consider the mean service time, which is required to complete the fixed point. From (33), noting that the complete transmission times $T^{s}, T_{r}^{b}$, and $T_{e}^{b}$ are constant we can express the mean service time for both schemes as

$$
\mathrm{E}\left[S^{i \in\{s, b\}}\right]=(1-\alpha)\left(\mathrm{E}\left[A_{r}^{i}\right]+T_{r}^{i}\right)+\alpha\left(\mathrm{E}\left[A_{e}^{i}\right]+T_{e}^{i}\right),
$$

where the expected values of the access delay $\mathrm{E}\left[A_{j}^{i}\right]$ can be calculated from (34) and (36).

Thus, based on (40), we can derive the mean service time in terms of $b$ and $\rho$. Now (2), (6), (10), (11), (13), (14) and (40) constitute a non-linear system of equations that can be solved iteratively to calculate $\rho, b, p$, and $\mathrm{E}[S]$. This completes the fixed point calculation of the direct collision probabilities. 
Each station will typically only have one message to send at a time, in which case $E[S]$ given by (40) is the total delay. However, if the load is unusually high, then the queueing delay can be calculated using the following calculations. This is the second place in which the Poisson assumption is used.

The total packet delay can be approximated by considering each node to be an $M / G / 1$ queue. Note that this is only an approximation, since the service time here depends on whether or not it arrives at an empty queue, whereas that is not the case in the M/G/1 model. In order to use the M/G/1 model, we require the variance of the service time. This can be obtained analogously to the mean.

From (33), noting that the complete transmission times $T^{s}, T_{r}^{b}$, and $T_{e}^{b}$ are constant, we can express the variance of the service time for both schemes as

$$
\begin{array}{r}
\operatorname{Var}\left[S^{i \in\{s, b\}}\right]=(1-\alpha)\left(\operatorname{Var}\left[A_{r}^{i}\right]+\left(\mathrm{E}\left[S^{i}\right]-\mathrm{E}\left[A_{r}^{i}\right]-T_{r}^{i}\right)^{2}\right) \\
+\alpha\left(\operatorname{Var}\left[A_{e}^{i}\right]+\left(\mathrm{E}\left[S^{i}\right]-\mathrm{E}\left[A_{e}^{i}\right]-T_{e}^{i}\right)^{2}\right)
\end{array}
$$

The variances of the access delays $\operatorname{Var}\left[A_{j}^{i}\right]$ in (41) can again be calculated from (34) and (36).

Using those values we can derive the mean queueing delay, $\mathrm{E}[Q]$, using the well-known result for the M/G/1 queue:

$$
\mathrm{E}[Q]=\frac{\lambda\left(\operatorname{Var}[S]+\mathrm{E}[S]^{2}\right)}{2(1-\lambda \mathrm{E}[S])}
$$

The mean total delay is then given by

$$
\mathrm{E}[D]=\mathrm{E}[Q]+\mathrm{E}[S]
$$

Recall that this is a conservative bound on the delay experienced by the application, which is the time until the packet is first received successfully.

\section{ViII. Performance Evaluation}

In this section, we investigate the proposed schemes using a combination of simulation and the model derived in the previous section. For simulation, we use the ns-2 simulator (version 2.28) [26], with the patch provided in [27], to simulate and obtain packet delay and PDR. We use a ring topology in the simulation where we place vehicles on a circle to avoid any unwanted boundary effects. The vehicles are placed randomly on the circle where the average inter-vehicle distance is a function of vehicle density, $\beta$. Each vehicle is setup to broadcast messages with packet size $P=400$ bytes and overall packet arrival rate $\lambda=10$ packets per second of both routine and event messages following a Poisson process. All the other DSRC related parameters are listed in Table I. 
TABLE I

DSRC SYSTEM PARAMETERS

\begin{tabular}{cc||cc}
\hline Parameter & Value & Parameter & Value \\
\hline $\mathrm{W}$ & 32 & Range, $R$ & $250 \mathrm{~m}$ \\
Slot size, $\sigma$ & $16 \mu \mathrm{s}$ & DIFS & $64 \mu \mathrm{s}$ \\
SIFS & $32 \mu \mathrm{s}$ & $\alpha$ & $0.1,0.5$ \\
Vehicle density, $\beta$ & $0.01-0.1 \mathrm{~m}^{-1}$ & Data rate, $R_{d}$ & $12 \mathrm{Mbps}$ \\
Packet arrival rate, $\lambda$ & $10 \mathrm{~s}^{-1}$ & Packet length, $P$ & 400 bytes \\
\hline
\end{tabular}

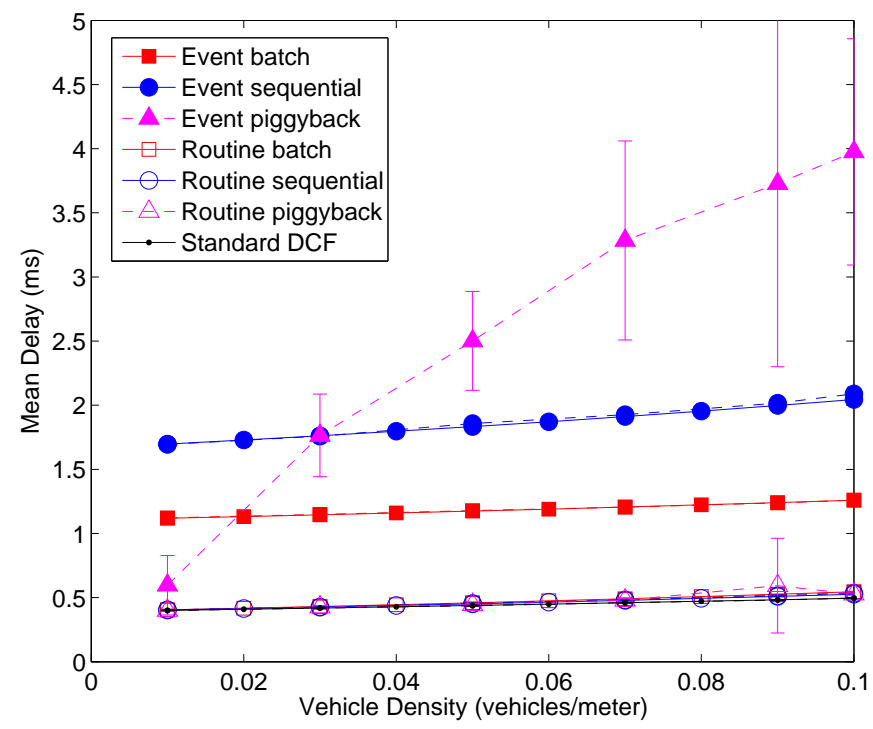

Fig. 1. Total delay until last transmission for the sequential, batch, standard DCF and piggybacked schemes with $\alpha=0.1$. Analytical results use solid lines, while simulation results use dashed lines. Only simulation results are given for the piggybacked scheme.

Before evaluating the overall benefits of the proposed schemes, we first validate the analytical model for the sequential and batch schemes by comparing the numerical results with the simulation for $m=3$ transmission attempts per packet. All simulation results are plotted with $95 \%$ confidence intervals.

Results in Figs 1 and 2 are generated assuming $10 \%$ of safety messages are event packets $(\alpha=0.1)$. Fig. 1 shows the mean of the total delay (43) as a function of vehicle density, $\beta$. Different curves are plotted for the delay of routine and event messages for the sequential and batch schemes, while the delay for standard DCF is represented by a single curve since DCF does not differentiate between routine and 


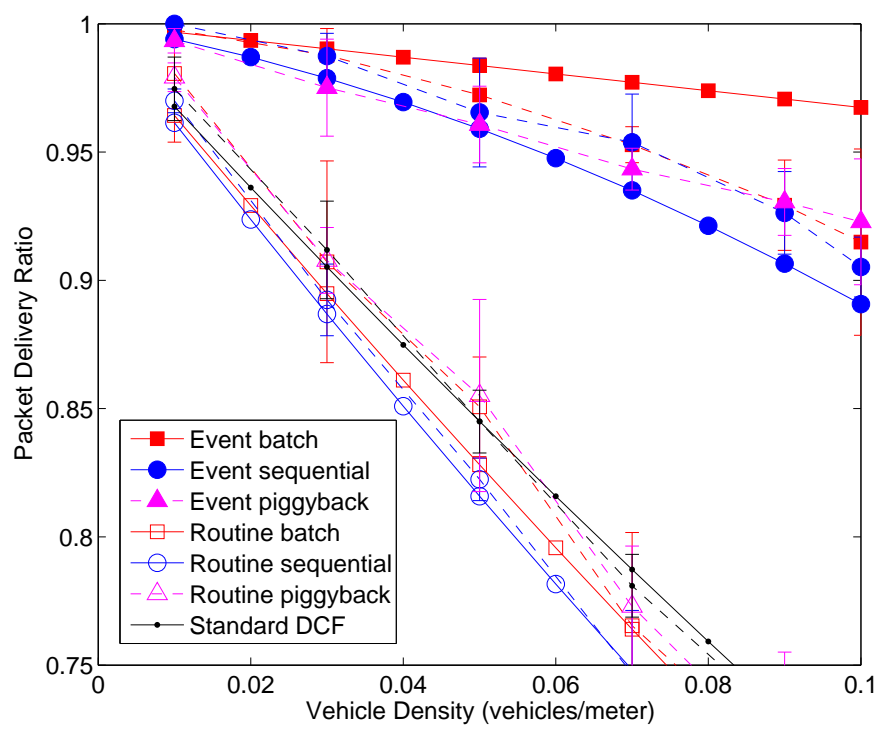

Fig. 2. PDR for the sequential, batch, standard DCF and piggybacked schemes with $\alpha=0.1$. Analytical results use solid lines, while simulation results use dashed lines. Only simulation results are given for the piggybacked scheme.

event messages. The analytical results are plotted using solid lines while the simulations results appear as dashed lines. Observe that the proposed analytical model agrees well with the simulation results. Simulation results for the piggybacked acknowledgement (PACK) scheme from [13] are also plotted for comparison, and we will return to this shortly.

The longest mean delay observed for our proposed sequential and batch schemes is around $2.1 \mathrm{~ms}$ which is well below the maximum delay constraint of $100 \mathrm{~ms}$ for safety applications [28]. This is despite the "total delay" for event packets being measured as the delay until the last copy is sent, rather than until the packet is first received; if the probability of success is high, then this will greatly over-estimate the delay penalty for using the retransmission schemes. Note also that the actual delays are more closely clustered around the mean for DSRC than for protocols with binary exponential backoff, and so it is unlikely that any packet incurs a delay near $100 \mathrm{~ms}$. In the plotted range, the mean delay increases almost linearly with the vehicle density. Also, the delays for routine safety packets for both sequential and batch schemes are similar. Under both schemes, the delay for event packets is greater than that for routine safety packets due to retransmissions, with the difference being greater for the sequential scheme due to the backoff between each transmission attempt.

We also compare our results with simulation results for the piggybacked acknowledgement (PACK) 


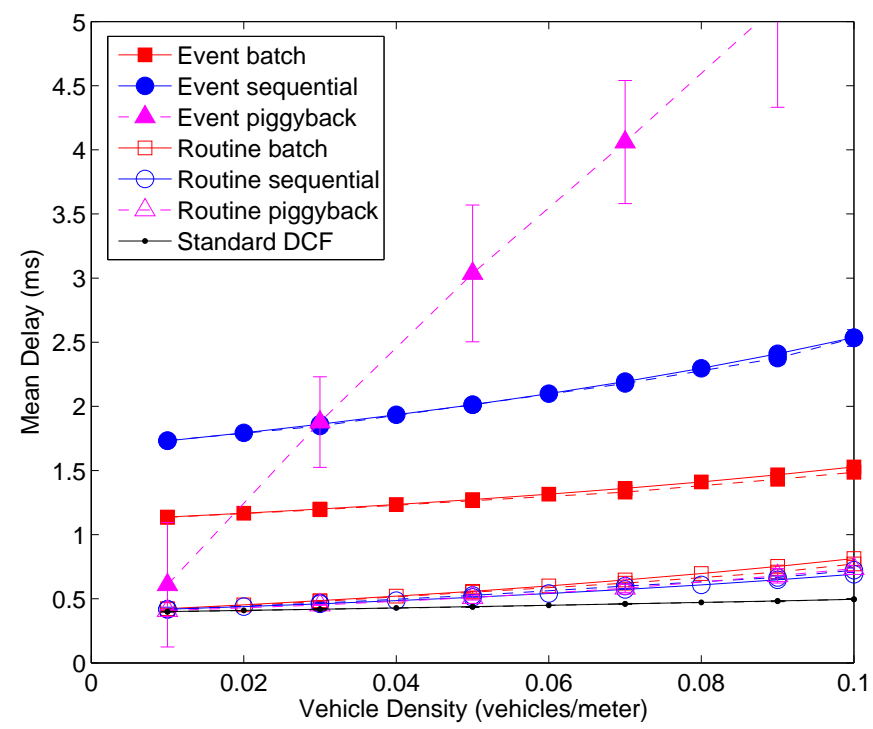

Fig. 3. Total delay until last transmission for the sequential, batch, standard DCF and piggybacked transmission schemes with $\alpha=0.5$

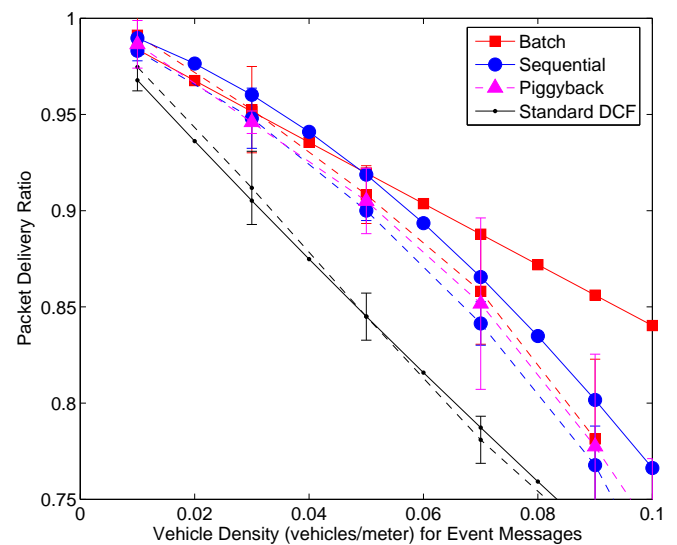

(a) Event messages

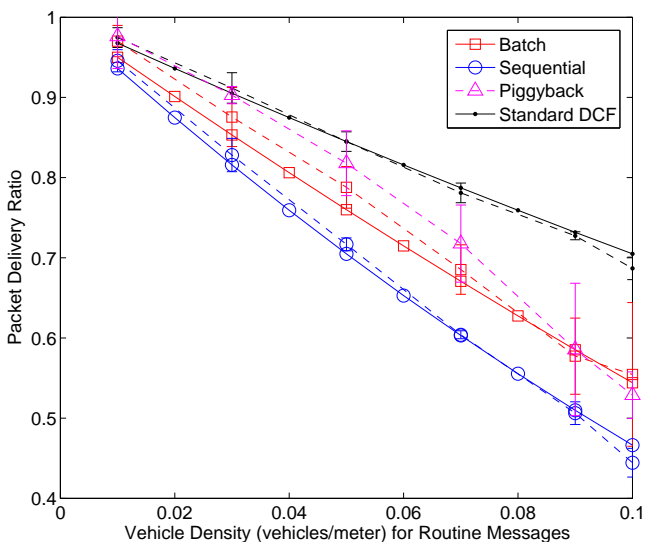

(b) Routine messages

Fig. 4. PDR for the sequential, batch, standard DCF and piggybacked transmission schemes with $\alpha=0.5$ 
protocol proposed in [13]. Note that in the original piggybacked protocol, a packet was retransmitted only if the fraction of recipients that did not acknowledge it was above a threshold. Herein we take that threshold to be 0 ; thus any packet suffering loss is simply retransmitted until either it is received by all intended receivers, or the number of retransmission reaches the maximum limit $m=3$. Fig. 1 shows that for the piggybacked retransmission scheme, the delay until the last transmission is significantly higher than both the sequential and batch schemes when the vehicle density is not low. This is because of the need to wait for the piggybacked feedback before each retransmission.

The packet delivery ratio is plotted in Fig. 2, where the analytical results are computed according to (27)-(31). The model matches reasonably well with the simulation results, except that the model overestimates the PDR for event messages in the batch scheme for high vehicle densities. The reason is that the model ignores the possibility of a batch colliding with multiple packets. The model could be extended to account for this, but at the expense of additional complexity. For both sequential and batch schemes, there is a significant improvement in PDR for event packets over routine packets. For example, the simulated PDR for event packets stays above $90 \%$ for all studied vehicle densities, while the PDR for routine packets drops to around $70 \%$ for high vehicle densities.

Figs. 3 and 4 show the mean of the total delay and the PDR for all schemes when $50 \%$ of safety messages are event packets $(\alpha=0.5)$. The increase in the proportion of event packets causes only a slight increase in the mean total delay; as before, the piggybacked scheme has the largest delay for moderate to high vehicle densities. However, there are significantly more collisions (i.e., lower PDR) for all schemes for the larger value of $\alpha$.

Figs. 2 and 4 show that the batch and sequential retransmission schemes perform similarly for event messages. The model captures this similarity at low loads, while at high loads, it over-estimates the PDR for event messages in the batch scheme as already mentioned. For routine messages, Figs. 2 and 4 show that the batch scheme gives a slightly higher PDR than the sequential scheme. The routine message PDR for sequential and batch schemes is less than that of standard DCF (particularly at $\alpha=0.5$ ), which is the penalty for improving the PDR of event messages through retransmissions.

The PDR for event messages in the piggybacked scheme is worse than that of the sequential and batch schemes at low and moderate vehicle densities, but slightly better at high vehicle density. The PDR for routine messages in the piggybacked scheme is similar to that of standard DCF at low vehicle density. This is because the piggybacked scheme retransmits fewer packets than the sequential and batch schemes. At high vehicle density, however, the PDR of routine messages drops and is comparable to the sequential and batch schemes. 


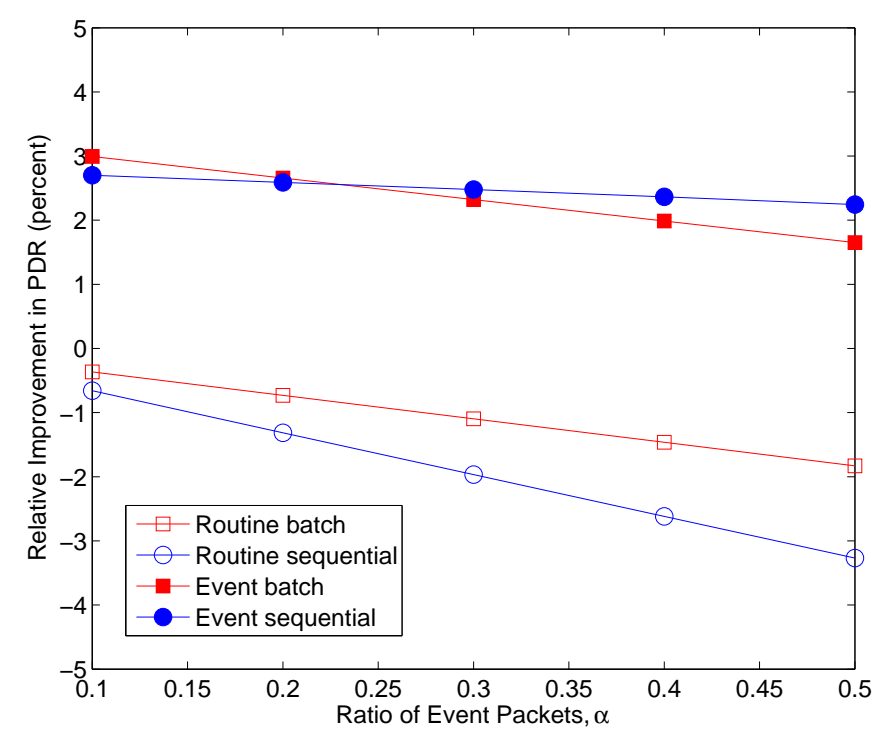

Fig. 5. Relative improvement of the PDR for the sequential and batch transmission schemes with $\beta=0.01$

We now use the model to evaluate the improvement due to both of the proposed schemes. Figs. 5 and 6 show the percentage improvement (or degradation) of the PDR between using single transmission and the two proposed retransmission schemes.

For low vehicle density $(\beta=0.01)$, the performance improvement in the PDR of event messages using retransmission is moderate as shown in Fig. 5. This is because at low traffic load, a single transmission would be sufficient to achieve adequate PDR performance for safety applications. On the other hand, at high vehicle density $(\beta=0.05)$ the relative improvement in PDR is higher. In particular, $10-15 \%$ improvement compared with using a single transmission is obtained for both retransmission schemes. The downside of using retransmission is a slight decrease in the PDR of routine safety messages, of up to $15 \%$, which would require that they be sent slightly more frequently to maintain a target arrival rate. The degradation due to batch retransmissions is less than that due to sequential transmissionssince it causes fewer partial collisions.

Fig. 7 shows the impact of the number retransmissions, $m$, on PDR for sequential scheme. In this figure, we numerically optimized $m$ in the range 1 to 5 . We observe that at light load it is beneficial to use high number of retransmission attempts, $m$. Note that the reduction in packet loss rate can be up to a factor of three at $\beta=0.05$ using $m=5$. However, at higher load, reducing $m$ helps by reducing channel load. 


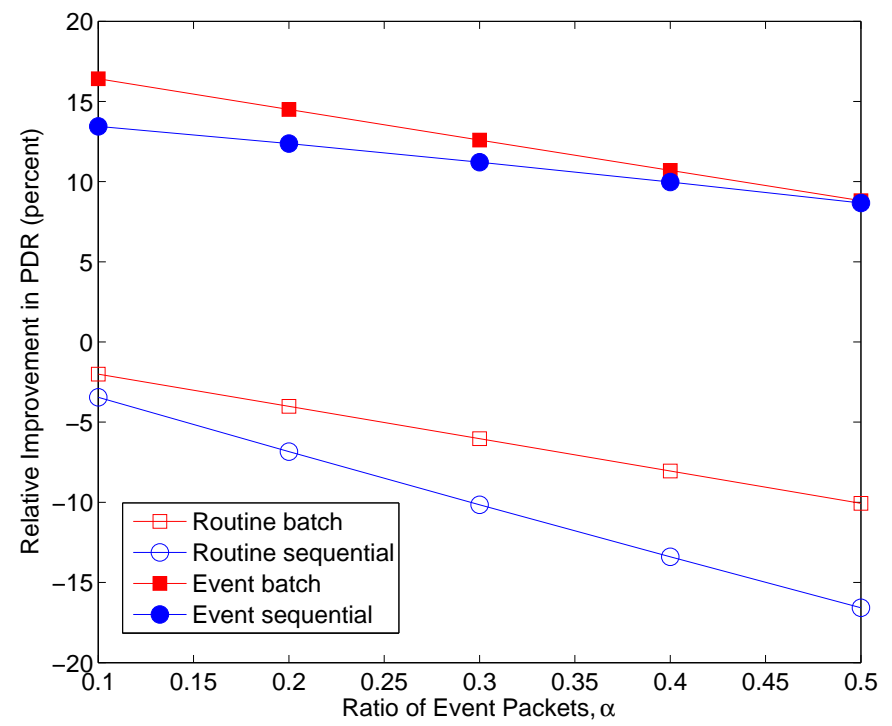

Fig. 6. Relative improvement of the PDR for the sequential and batch transmission schemes with $\beta=0.05$

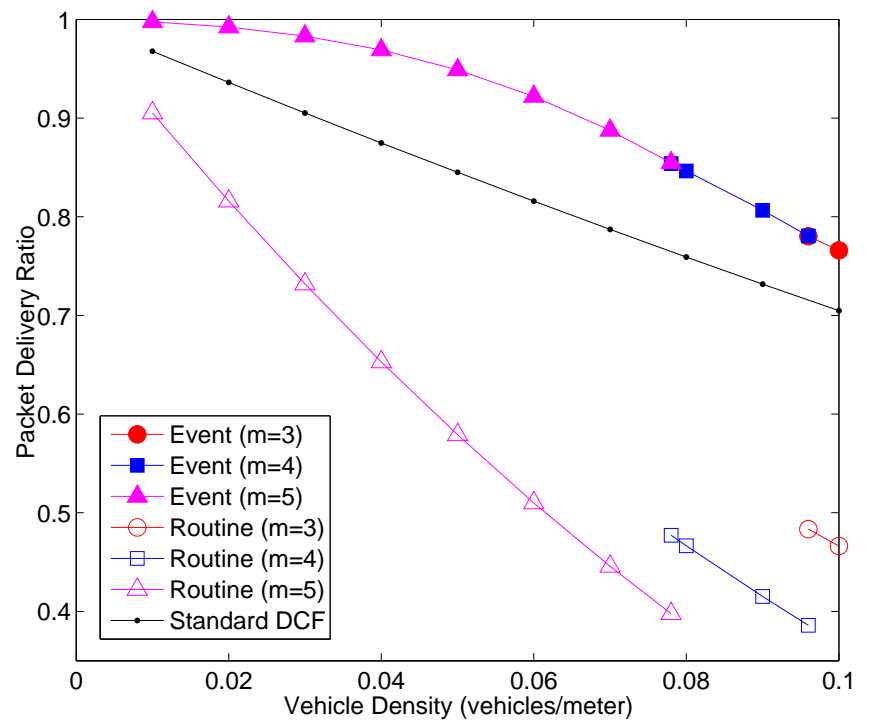

Fig. 7. The PDR for the sequential scheme with optimal $m \in[1,5]$ and $\alpha=0.5$ 


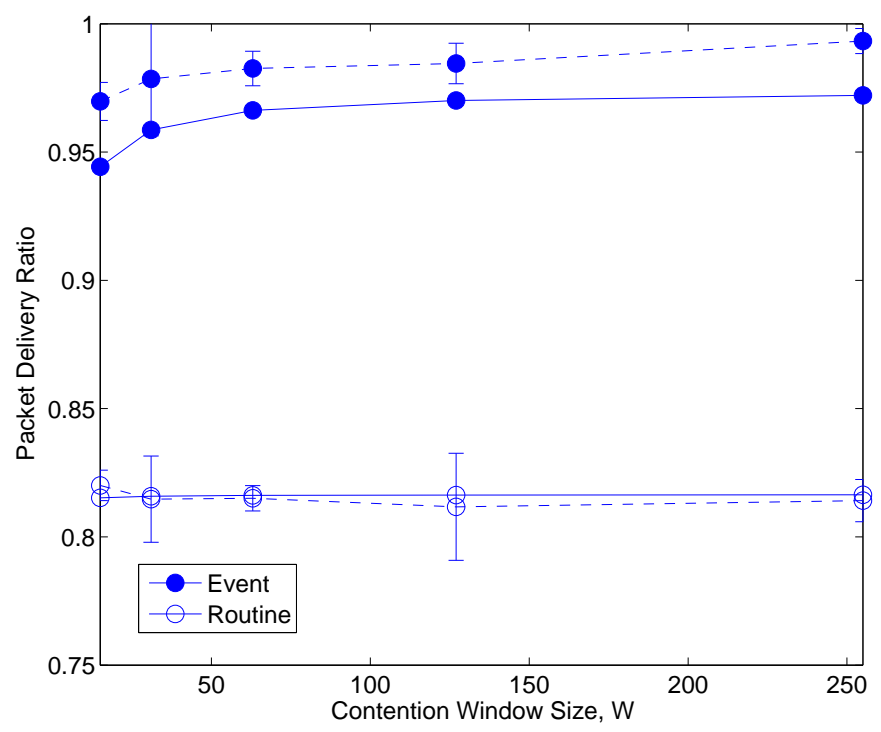

Fig. 8. PDR for the sequential scheme with with varying contention window, $W$ for $\beta=0.05$ and $\alpha=0.1$

To show the influence of the contention window size $W$, we plot the PDR for the sequential scheme against different $W$ in Fig. 8. For event packets, the PDR improves with increased $W$ since the larger space between retransmissions increases the temporal diversity. Although the model is somewhat conservative, it captures the large gap between the routine and event packets, and also the trend in PDR as $W$ increases. The performance of the batch scheme can be expected to be less sensitive to the contention window size, $W$, since it does not affect the diversity of the retransmissions.

\section{CONCLUSION}

In this paper, we have described two ways to incorporate retransmission into the broadcast mode of the 802.11 DCF and EDCA protocols in cooperative collision avoidance (CCA) applications, to blindly retransmit important safety packets. Both the proposed sequential and batch retransmission schemes improves the packet delivery ratio of the important messages by up to $15 \%$ when $10 \%$ of packets are important. This comes at a cost of reducing the PDR of routine packets by less than $10 \%$, and delay well within acceptable limits. The batch scheme, in which three copies of the packet are sent back-toback, slightly outperforms the sequential scheme at high load. However, the current $802.11 \mathrm{p}$ standard disallows the TXOP parameter for ad hoc transmissions, which are used by broadcasts. Based on the performance of the batch scheme, which uses that parameter, we recommend that it should be reinstated. 
The sequential scheme, in which there is a random backoff between retransmissions, could be further improved by increasing the spacing between retransmissions to increase the temporal diversity. This is the subject of ongoing evaluation.

We have also developed analytical models of the two schemes, and verified them against ns- 2 simulations. Future work will refine the batch model to consider multiple packet collisions, and use these models to determine the conditions under which batch retransmissions outperform sequential transmissions.

\section{REFERENCES}

[1] H. Hartenstein and K. P. Laberteaux, "A tutorial survey on vehicular ad hoc networks," IEEE Communications Magazine, vol. 46, no. 6, pp. 164-171, June 2008.

[2] F. Borgonovo, A. Capone, M. Cesana, and L. Fratta, "ADHOC MAC: new MAC architecture for ad hoc networks providing efficient and reliable point-to-point and broadcast services," Wireless Networks, vol. 10, no. 4, pp. 359-366, 2004.

[3] M. I. Hassan, H. L. Vu, T. Sakurai, L. L. H. Andrew, and M. Zukerman, "Effect of retransmissions on the performance of the IEEE 802.11 MAC protocol for DSRC,' in Proceedings of the IEEE Vehicular Networking Conference (VNC '10), Dec 2010.

[4] IEEE 802.11p, Part 11: Wireless LAN Medium Access Control (MAC) and Physical Layer (PHY) specifications: Amendment 6: Wireless Access in Vehicular Environments, IEEE Std., June 2010.

[5] ASTM E2213-03, Standard Specification for Telecommunications and Information Exchange Between Roadside and Vehicle Systems $-5 \mathrm{GHz}$ Band Dedicated Short Range Communications (DSRC) Medium Access Control (MAC) and Physical Layer (PHY) Specifications, ASTM Std., September 2003.

[6] X. Chen, H. H. Refai, and X. Ma, "A quantitative approach to evaluate DSRC highway inter-vehicle safety communication," in Proceedings of the IEEE Global Telecommunications Conference (GLOBECOM '07), November 2007, pp. 151-155.

[7] M. Demirbas and S. Balachandran, "Robcast: A singlehop reliable broadcast protocol for wireless sensor networks," in Proceedings of the International Conference on Distributed Computing Systems Workshops (ICDCSW '07), June 2007, pp. $54-54$.

[8] K. Tang and M. Gerla, "Random access MAC for efficient broadcast support in ad hoc networks," in Proceedings of the IEEE Wireless Communications and Networking Conference (WCNC '00), vol. 1, 2000, pp. 454-459.

[9] G. Korkmaz, E. Ekici, F. Özgüner, and U. Özgüner, "Urban multi-hop broadcast protocol for inter-vehicle communication systems," in Proceedings of the 1st ACM International Workshop on Vehicular Ad Hoc Networks (VANET '04), 2004, pp. 76-85.

[10] J. Sobrinho and A. Krishnakumar, "Quality-of-service in ad hoc carrier sense multiple access wireless networks," IEEE Journal on Selected Areas in Communications, vol. 17, no. 8, pp. 1353-1368, Aug 1999.

[11] K. Tang and M. Gerla, "MAC reliable broadcast in ad hoc networks," in Proceedings of the IEEE Military Communications Conference (MILCOM '01), vol. 2, 2001, pp. 1008-1013 vol.2.

[12] D. Jiang, V. Taliwal, A. Meier, W. Holfelder, and R. Herrtwich, "Design of $5.9 \mathrm{GHz}$ DSRC-based vehicular safety communication," IEEE Wireless Communications, vol. 13, no. 5, pp. 36-43, October 2006.

[13] D. Jiang, M. Bogdanovic, and L. Delgrossi, "Piggybacked acknowledgement for reception assessment in a pervasive broadcasting system," in Proceedings of the Eighth International Symposium on Autonomous Decentralized Systems (ISADS '07), 2007, pp. 387-393. 
[14] Q. Xu, R. Segupta, D. Jiang, and D. Chrysler, "Design and analysis of highway safety communication protocol in 5.9 GHz dedicated short range communication spectrum," in Proceedings of the 57th IEEE Vehicular Technology Conference (VTC '03-Spring), vol. 4, April 2003, pp. 2451-2455 vol.4.

[15] Q. Xu, T. Mak, J. Ko, and R. Sengupta, "Medium access control protocol design for vehicle-vehicle safety messages," IEEE Transactions on Vehicular Technology, vol. 56, no. 2, pp. 499-518, March 2007.

[16] G. Bianchi, "Performance analysis of the IEEE 802.11 distributed coordination function," IEEE Journal on Selected Areas in Communications, vol. 18, no. 3, pp. 535-547, March 2000.

[17] D. Malone, K. Duffy, and D. Leith, "Modeling the 802.11 distributed coordination function in nonsaturated heterogeneous conditions,” IEEE/ACM Transactions on Networking, vol. 15, no. 1, pp. 159-172, February 2007.

[18] O. Tickoo and B. Sikdar, "Queueing analysis and delay mitigation in IEEE 802.11 random access MAC based wireless networks," in Proc. INFOCOM, vol. 2, March 2004, pp. 1404-1413.

[19] A. Rao, A. Kherani, and A. Mahanti, "Performance evaluation of 802.11 broadcasts for a single cell network with unsaturated nodes," in Proceedings of the 7th international IFIP-TC6 Networking Conference on Ad Hoc and Sensor Networks, Wireless Networks, Next Generation Internet (NETWORKING '08). Berlin, Heidelberg: Springer-Verlag, 2008, pp. 836-847.

[20] R. Oliveira, L. Bernardo, and P. Pinto, "Performance analysis of the IEEE 802.11 distributed coordination function with unicast and broadcast traffic," in Proceedings of the IEEE International Symposium on Personal, Indoor and Mobile Radio Communications (PIMRC), 2006.

[21] A. Tsertou and D. Laurenson, "Revisiting the hidden terminal problem in a CSMA/CA wireless network," IEEE Transactions on Mobile Computing, vol. 7, no. 7, pp. 817-831, July 2008.

[22] L. Qiu, Y. Zhang, F. Wang, M. K. Han, and R. Mahajan, “A general model of wireless interference," in Proceedings of the 13th annual ACM international conference on Mobile computing and networking (MobiCom '07), ser. MobiCom '07. New York, NY, USA: ACM, 2007, pp. 171-182.

[23] N. Abramson, "The Aloha system: another alternative for computer communications," in Proceedings of the Joint Computer Conference (AFIPS '70-Fall). New York, NY, USA: ACM, 1970, pp. 281-285.

[24] M. Green, "How Long Does It Take to Stop? Methodological Analysis of Driver PerceptionBrake Times," Transportation Human Factors, vol. 2, no. 3, pp. 195-216, 2000. [Online]. Available: http://www.tandfonline.com/doi/abs/10.1207/STHF0203_1

[25] F. Bai, D. D. Stancil, and H. Krishnan, "Toward understanding characteristics of dedicated short range communications (DSRC) from a perspective of vehicular network engineers," in Proceedings of the Sixteenth Annual International Conference on Mobile Computing and Networking (MobiCom '10), ser. MobiCom '10. New York, NY, USA: ACM, 2010, pp. 329-340. [Online]. Available: http://doi.acm.org/10.1145/1859995.1860033

[26] “The network simulator ns-2." [Online]. Available: http://www.isi.edu/nsnam/ns/

[27] F. Schmidt-Eisenlohr, J. Letamendia-Murua, M. Torrent-Moreno, and H. Hartenstein, "Bug fixes on the IEEE 802.11 DCF module of the Network Simulator ns-2.28," Department of Computer Science, University of Karlsruhe, Tech. Rep. TR-2006-1, January 2006.

[28] T. K. Mak, K. P. Laberteaux, and R. Sengupta, "A multi-channel VANET providing concurrent safety and commercial services," in Proceedings of the 2nd ACM International Workshop on Vehicular Ad Hoc Networks (VANET '05), ser. VANET '05. New York, NY, USA: ACM, 2005, pp. 1-9. 